\title{
THE CHROMATIC POLYNOMIAL OF A GRAPH
}

\author{
D. H. LEHMER \\ Dedicated to the memory of Ernst G. Straus
}

\begin{abstract}
The problem of which polynomials occur as the vertex coloring of a graph is studied. The results are complete for graphs with fewer than seven vertices.
\end{abstract}

The vertex coloring of a graph is an assignment of colors to the vertices of the graph in such a way that no edge has its end points colored the same. If $\lambda$ colors are available the number of ways that a graph $G$ can be so colored is denoted by $P(G, \lambda)$. This function is a monic polynomial of degree $V$ with integer coefficients, where $V$ is the number of vertices. The smallest positive integer $\lambda$ for which $P(G, \lambda)>0$ is called the chromatic number of $G$. For example, the chromatic polynomial of a triangle is easily seen to be

$$
\lambda^{3}-3 \lambda^{2}+2 \lambda=\lambda(\lambda-1)(\lambda-2)
$$

while that for the cube is

$$
\begin{aligned}
& \lambda^{8}-12 \lambda^{7}+66 \lambda^{6}-214 \lambda^{5}+441 \lambda^{4}-572 \lambda^{3}+423 \lambda^{2}-133 \lambda \\
& \quad=\lambda(\lambda-1)\left(\lambda^{6}-11 \lambda^{5}+55 \lambda^{4}-159 \lambda^{3}+282 \lambda^{2}-290 \lambda+133\right) .
\end{aligned}
$$

Thus the cube can be colored in two colors in just two ways while the triangle requires three colors and then can be colored in six ways.

An outstanding problem in graph theory is to decide whether a given polynomial is the chromatic polynomial of some graph. It is the purpose of this paper to look into this question. A complete account is given for graphs with fewer than seven vertices. A statistical sample of 100 of the graphs with 10 vertices is also discussed.

Suppose the given polynomial is

$$
P(\lambda)=\lambda^{v}-a_{1} \lambda^{v-1}+a_{2} \lambda^{v-2}+\cdots+(-1)^{v-1} a_{v-1} \lambda+(-1)^{v} a_{v} .
$$

Then $v$ must be the number of vertices of the proposed graph $G$. Secondly it is known that $a_{1}$ is the number of edges of $G$. Furthermore, it is known that $a_{i} \geq 0$. Since a graph with at least one edge cannot be colored in less than two colors, it follows that $P(\lambda)$ is divisible by $\lambda(\lambda-1)$. In particular 
$a_{v}$ must be zero. If we continue to look for the roots $2,3, \ldots$ and remove these to whatever multiplicities they occur, we can write $P(\lambda)$ in the form

$$
\begin{array}{r}
P(\lambda)=\lambda^{m_{0}}(\lambda-1)^{m_{1}}(\lambda-2)^{m_{2}} \cdots(\lambda-k+1)^{m_{k-1}} R(\lambda) \\
\quad\left(m_{i} \geq 1\right),
\end{array}
$$

where $R(k) \neq 0$. We call $R(\lambda)$ the residual quotient of $P(\lambda)$. If there is a graph $G$ for which

$$
P(\lambda)=P(G, \lambda)
$$

then a necessary condition on $R(\lambda)$ is that $R(\lambda)$ have no rational root. In fact, since $R(\lambda)$ is monic, its rational roots are integers and indeed positive integers because $a_{i} \geq 0$. Furthermore its integer roots, if any, are $>k$ by definition of $R(\lambda)$. If $R(n)=0$ for some integer $n>k$ then $G$ could not be colored in $n$ colors but could be colored in $k<n$ colors. This is a contradiction.

The number $k$ defined by (2) is, of course, the chromatic number of $G$.

If we are examining a set of graphs $G$ to discover new residual quotients $R(\lambda)$, our search will be considerably reduced by using the following four theorems. Although these are known theorems (see for example Ore [1]), their proofs are sketched and a few comments are given.

THEOREM 1. Suppose that $G$ is not connected so that there exist two graphs $G_{1}$ and $G_{2}$ such that

$$
G=G_{1} \cup G_{2} \text { and } G_{1} \cap G_{2}=0 .
$$

Then

$$
P(G, \lambda)=P\left(G_{1}, \lambda\right) \cdot P\left(G_{2}, \lambda\right) .
$$

Proof. Color each of $G_{1}$ and $G_{2}$ separately. Since they do not intersect, the theorem follows from a fundamental combinatorial principle.

Two facts follow from Theorem 1:

(a) The set of all chromatic polynomials is closed under multiplication.

(b) In looking for new residual quotients we may confine our attention to connected graphs.

THEOREM 2. Let $G$ be the union of two graphs $G_{1}$ and $G_{2}$ that have a single vertex in common. Then

$$
P(G, \lambda)=\frac{P\left(G_{1}, \lambda\right) P\left(G_{2}, \lambda\right)}{\lambda} .
$$


Proof. Let $V$ be the common vertex. If we color $G_{1}$ and $G_{2}$ separately $V$ will be colored the same way only one time in $\lambda$. Such graphs can be omitted in our search for new residual quotients. Graphs with "whiskers", that is vertices of valence 1 , belong to this class. The chromatic polynomial of a graph with $n$ whiskers is divisible by $(\lambda-1)^{n+1}$.

THEOREM 3. Let $G$ have an edge $E$ whose destruction would cause $G$ to become two disconnected graphs $G_{1}$ and $G_{2}$. Then

$$
P(G, \lambda)=\frac{(\lambda-1) P\left(G_{1}, \lambda\right) P\left(G_{2}, \lambda\right)}{\lambda} .
$$

Proof. If we color $G_{1}$ and $G_{2}$ separately the two ends of $E$ connecting $G_{1}$ and $G_{2}$ will be colored differently $\lambda-1$ times out of $\lambda$.

THEOREM 4. Let $G$ be the union of two graphs $G_{1}$ and $G_{2}$ that have only one edge in common. Then

$$
P(G, \lambda)=\frac{P\left(G_{1}, \lambda\right) P\left(G_{2}, \lambda\right)}{[\lambda(\lambda-1)]} .
$$

Proof. Let $A$ and $B$ be the vertices of the common edge. By Theorem 1, the colors of $A$ and $B$ being specified, the rest of $G_{2}$ can be colored in

$$
Q=\frac{P\left(G_{2}, \lambda\right)}{[\lambda(\lambda-1)]}
$$

ways. To color $G$ we may first color $G_{1}$ in $P\left(G_{1}, \lambda\right)$ ways and then color $G_{2}$ in $Q$ ways.

A common corollary of this theorem is the case of the "attached triangle" in which there is a bivalent vertex $V$ whose other vertices are adjacent vertices of $G$. In this case,

$$
P(G, \lambda)=P\left(G_{1}, \lambda\right)(\lambda-2) .
$$

If $G$ is disconnected or nearly disconnected in the sense of Theorems 2, 3 and 4 it is clear that $G$ will not produce a "new" residual quotient, that is, its residual quotient is the residual quotient of a simpler graph. Graphs that don't satisfy the hypotheses of Theorems 1 to 4 we call sturdy. In the following table we give the number $N$ of graphs, the number $N_{c}$ of connected graphs, and the number $N_{s}$ of sturdy graphs with $V$ vertices for $1 \leq v \leq 6$. 


\begin{tabular}{rrrr}
\hline$V$ & $N$ & $N_{c}$ & $N_{s}$ \\
\hline 1 & 1 & 1 & 0 \\
2 & 2 & 1 & 0 \\
3 & 4 & 2 & 0 \\
4 & 11 & 6 & 2 \\
5 & 34 & 21 & 5 \\
6 & 156 & 112 & 26 \\
Totals & $\underline{208}$ & $\overline{143}$ & $\frac{33}{}$ \\
\hline
\end{tabular}

Hence by examining only 33 graphs with $\leq 6$ vertices it is possible to find all the residual quotients of 208 graphs. These may be listed in lexicographical order, along with their discriminants as follows. There are only 21 of these polynomials since several non-isomorphic graphs have the same residual quotients.

$\begin{array}{lc}\quad \text { Residual Quotient } & \text { Discriminant } \\ \lambda^{2}-2 \lambda+2 & -4 \\ \lambda^{2}-3 \lambda+3 & -3 \\ \lambda^{2}-3 \lambda+4 & -7 \\ \lambda^{2}-4 \lambda+5 & -4 \\ \lambda^{2}-4 \lambda+6 & -8 \\ \lambda^{2}-5 \lambda+7 & -3 \\ \lambda^{2}-5 \lambda+8 & -7 \\ \lambda^{2}-6 \lambda+10 & -4 \\ \lambda^{3}-4 \lambda^{2}+7 \lambda-5 & -23 \\ \lambda^{3}-5 \lambda^{3}+9 \lambda-7 & -44 \\ \lambda^{3}-5 \lambda^{2}+10 \lambda-7 & -23 \\ \lambda^{3}-6 \lambda^{2}+13 \lambda-11 & -31 \\ \lambda^{3}-6 \lambda^{2}-14 \lambda-11 & -59 \\ \lambda^{3}-6 \lambda^{2}+14 \lambda-13 & -59 \\ \lambda^{3}-7 \lambda^{2}+18 \lambda-17 & -23 \\ \lambda^{3}-7 \lambda^{2}+19 \lambda-19 & -76 \\ \lambda^{3}-8 \lambda^{2}+23 \lambda-23 & -23 \\ \lambda^{3}-9 \lambda^{2}+28 \lambda-29 & -31 \\ \lambda^{4}-5 \lambda^{3}+10 \lambda^{2}-10 \lambda+5 & 125 \\ \lambda^{4}-7 \lambda^{3}+21 \lambda^{2}-30 \lambda+17 & 257 \\ \lambda^{4}-8 \lambda^{3}+28 \lambda^{2}-47 \lambda+31 & 2597\end{array}$


There are 1044 graphs with 7 vertices; 853 or $82 \%$ of these are connected graphs. A random sample of 100 of these graphs showed 20 to be sturdy.

It was decided to take a random sample of 100 graphs for the $12,005,168$ graphs with 10 vertices (of which 11,716,571 are connected). These were fully analyzed for their residual quotients. Of these 100 graphs 64 were sturdy. We list below those residual quotients not found in the above tabulation together with their discriminants.

$\begin{array}{lr}\quad \text { Residual Quotient } & \text { Discriminant } \\ \lambda^{2}-5 \lambda+9 & -11 \\ \lambda^{2}-6 \lambda+11 & -8 \\ \lambda^{3}-8 \lambda+25 \lambda-29 & -199 \\ \lambda^{3}-10 \lambda^{2}-35 \lambda-43 & -23 \\ \lambda^{3}-11 \lambda^{2}+42 \lambda-55 & -23 \\ \lambda^{3}-11 \lambda^{2}+43 \lambda-58 & -107 \\ \lambda^{4}-9 \lambda^{3}+34 \lambda^{2}-69 \lambda+50 & 2836 \\ \lambda^{4}-10 \lambda^{3}+41 \lambda^{2}-80 \lambda+61 & 144 \\ \lambda^{4}-10 \lambda^{3}+42 \lambda^{2}-86 \lambda+72 & 5584 \\ \lambda^{4}-11 \lambda^{3}+51 \lambda^{2}-117 \lambda+109 & 16357 \\ \lambda^{4}-12 \lambda^{3}+56 \lambda^{2}-121 \lambda+101 & 1371 \\ \lambda^{4}-12 \lambda^{3}+57 \lambda^{2}-126 \lambda+112 & 3136 \\ \lambda^{4}-12 \lambda^{3}+58 \lambda^{2}-130 \lambda+114 & 6224 \\ \lambda^{4}-12 \lambda^{3}+60 \lambda^{2}-145 \lambda+143 & 23909 \\ \lambda^{4}-13 \lambda^{3}+65 \lambda^{2}-149 \lambda+131 & 1963 \\ \lambda^{4}-16 \lambda^{3}+100 \lambda^{2}-288 \lambda+322 & 2048 \\ \lambda^{4}-17 \lambda^{3}+112 \lambda^{2}-339 \lambda+398 & 1929\end{array}$

In addition to the above there were

31 polynomials of degree 5

27 polynomials of degree 6

13 polynomials of degree 7 .

As to the distribution of chromatic numbers among the 100 random graphs: 29 graphs need three colors and 56 need four colors and 15 need five colors.

The number of edges range from 14 to 35 and are distributed as follows. 


$\begin{array}{cccr}\begin{array}{c}\text { Number of } \\ \text { Edges }\end{array} & \begin{array}{c}\text { Number of } \\ \text { Graphs }\end{array} & \begin{array}{c}\text { Number of } \\ \text { Edges }\end{array} & \begin{array}{c}\text { Number of } \\ \text { Graphs }\end{array} \\ 14 & 3 & 23 & 11 \\ 15 & 1 & 24 & 17 \\ 16 & 1 & 25 & 10 \\ 17 & 6 & 26 & 5 \\ 18 & 5 & 27 & 6 \\ 19 & 6 & 28 & 3 \\ 20 & 8 & 29 & 3 \\ 21 & 6 & 30 & 1 \\ 22 & 7 & 35 & 1\end{array}$

76 of the 100 graphs had Hamiltonian circuits.

The roots of $R(\lambda)$ are for the most part tightly clustered. This is shown by the smallness of its discriminant. No cubic polynomial has a discriminant less than -23 and six residual quotients have this minimum value. However there are graphs whose residual quotient have quite large discriminants. For example, the discriminant for the cube graph is $-39,069,367$.

Of course, all these results were obtained electronically. We conclude this report by giving a very brief account of the algorithms used.

In the first place, all graphs are handled by means of their adjacency matrix $\left\{a_{i J}\right\}_{v \times v}$ where

$$
a_{i j}= \begin{cases}1 & \text { if vertex } i \text { is adjacent to vertex } j \\ 0 & \text { otherwise. }\end{cases}
$$

This is a symmetric matrix with 0 's on its diagonal. Those elements which lie above the main diagonal can be assigned arbitrarily to determine an arbitrary graph. To make a random choice of the $i$ th row of this upper triangular matrix we generate a large random integer, extract $V$ binary digits from its center and decapitate $i$ of its digits. This leaves a $V-i$-digit random binary number for the $i$ th row. The matrix is now made symmetric.

To find $P(G, \lambda)$ we use the following.

THEOREM 5. Let $G$ be a graph with an edge $E$. Let the endpoints of $E$ be $V_{1}$ and $V_{2}$. Let $G^{\prime}$ be the result of destroying $E$ (but not $V_{1}$ or $V_{2}$ ). Let $G^{\prime \prime}$ be the graph obtained from $G^{\prime}$ by letting $V_{1}$ coincide with $V_{2}$. Then

$$
P(G, \lambda)=P\left(G^{\prime}, \lambda\right)-P\left(G^{\prime \prime}, \lambda\right) .
$$


Proof. We can color $G^{\prime}$ in $\lambda$ colors by coloring $G$ properly and then coloring $G$ improperly so that the vertices $V_{1}$ and $V_{2}$ have the same color. This last operation is equivalent to coloring $G^{\prime \prime}$ properly. That is,

$$
P\left(G^{\prime}, \lambda\right)=P(G, \lambda)+P\left(G^{\prime \prime}, \lambda\right) .
$$

To use Theorem 5 in finding the chromatic polynomial of a given graph $G$ with $E$ edges one proceeds as follows.

First, one replaces $G$ by the two graphs $G^{\prime}$ and $G^{\prime \prime}$ and sends them to two stores, $S_{+}$and $S_{-}$. Each of them has at most $E-1$ edges. Next, one processes in the same way the graphs in $S_{+}$and $S_{-}$, being careful to place the output graphs, which now have at most $E-2$ edges, in the appropriate storage.

One proceeds in this way until, in $E$ steps, there are no edges left in any of the graphs.

A simple counting process collects the coefficients of the chromatic polynomial of $G$.

\section{REFERENCE}

[1] O. Ore, Theory of Graphs, Amer. Math. Soc. Colloquium Publications, Vol. 38 (Providence, 1962).

Received June 7, 1984.

UNIVERSITY OF CALIFORNIA

BERKELEY, CA 94720 
